\title{
Learning Semantic Classes for Word Sense Disambiguation
}

\author{
Upali S. Kohomban Wee Sun Lee \\ Department of Computer Science \\ National University of Singapore \\ Singapore, 117584 \\ \{upalisat, leews\}@ comp.nus.edu.sg
}

\begin{abstract}
Word Sense Disambiguation suffers from a long-standing problem of knowledge acquisition bottleneck. Although state of the art supervised systems report good accuracies for selected words, they have not been shown to be promising in terms of scalability. In this paper, we present an approach for learning coarser and more general set of concepts from a sense tagged corpus, in order to alleviate the knowledge acquisition bottleneck. We show that these general concepts can be transformed to fine grained word senses using simple heuristics, and applying the technique for recent SENSEVAL data sets shows that our approach can yield state of the art performance.
\end{abstract}

\section{Introduction}

Word Sense Disambiguation (WSD) is the task of determining the meaning of a word in a given context. This task has a long history in natural language processing, and is considered to be an intermediate task, success of which is considered to be important for other tasks such as Machine Translation, Language Understanding, and Information Retrieval.

Despite a long history of attempts to solve WSD problem by empirical means, there is not any clear consensus on what it takes to build a high performance implementation of WSD. Algorithms based on Supervised Learning, in general, show better performance compared to unsupervised systems. But they suffer from a serious drawback: the difficulty of acquiring considerable amounts of training data, also known as knowledge acquisition bottleneck. In the typical setting, supervised learning needs training data created for each and every polysemous word; $\mathrm{Ng}$ (1997) estimates an effort of 16 personyears for acquiring training data for 3,200 significant words in English. Mihalcea and Chklovski (2003) provide a similar estimate of an 80 person-year effort for creating manually labelled training data for about 20,000 words in a common English dictionary.

Two basic approaches have been tried as solutions to the lack of training data, namely unsupervised systems and semi-supervised bootstrapping techniques. Unsupervised systems mostly work on knowledge-based techniques, exploiting sense knowledge encoded in machine-readable dictionary entries, taxonomical hierarchies such as WORDNET (Fellbaum, 1998), and so on. Most of the bootstrapping techniques start from a few 'seed' labelled examples, classify some unlabelled instances using this knowledge, and iteratively expand their knowledge using information available within newly labelled data. Some others employ hierarchical relatives such as hypernyms and hyponyms.

In this work, we present another practical alternative: we reduce the WSD problem to a one of finding generic semantic class of a given word instance. We show that learning such classes can help relieve the problem of knowledge acquisition bottleneck.

\subsection{Learning senses as concepts}

As the semantic classes we propose learning, we use WORDNET lexicographer file identifiers corre- 
sponding to each fine-grained sense. By learning these generic classes, we show that we can reuse training data, without having to rely on specific training data for each word. This can be done because the semantic classes are common to words unlike senses; for learning the properties of a given class, we can use the data from various words. For instance, the noun crane falls into two semantic classes ANIMAL and ARTEFACT. We can expect the words such as pelican and eagle (in the bird sense) to have similar usage patterns to those of ANIMAL sense of crane, and to provide common training examples for that particular class.

For learning these classes, we can make use of any training example labelled with WORDNET senses for supervised WSD, as we describe in section 3.1.

Once the classification is done for an instance, the resulting semantic classes can be transformed into finer grained senses using some heuristical mapping, as we show in the next sub section. This would not guarantee a perfect conversion because such a mapping can miss some finer senses, but as we show in what follows, this problem in itself does not prevent us from attaining good performance in a practical WSD setting.

\subsection{Information loss in coarse grained senses}

As an empirical verification of the hypothesis that we can still build effective fine-grained sense disambiguators despite the loss of information, we analyzed the performance of a hypothetical coarse grained classifier that can perform at $100 \%$ accuracy. As the general set of classes, we used WORDNET unique beginners, of which there are 25 for nouns, and 15 for verbs.

To simulate this classifier on SENSEVAL English all-words tasks' data (Edmonds and Cotton, 2001; Snyder and Palmer, 2004), we mapped the finegrained senses from official answer keys to their respective beginners. There is an information loss in this mapping, because each unique beginner can typically include more than one sense. To see how this 'classifier' fares in a fine-grained task, we can map the 'answers' back to WORDNET fine-grained senses by picking up the sense with the lowest sense number that falls within each unique beginner. In principal, this is the most likely sense within the class, because WORDNET senses are said to be

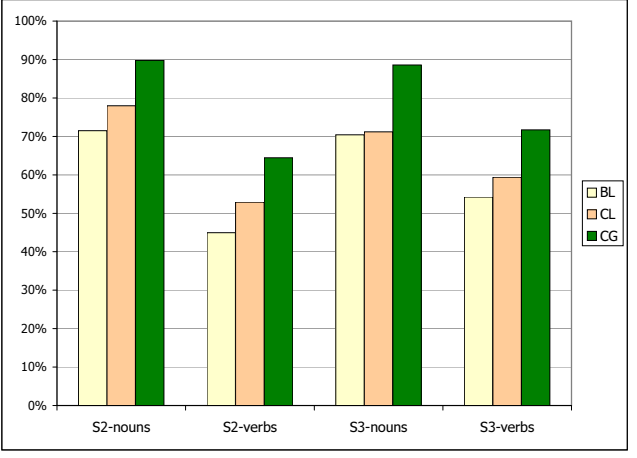

Figure 1: Performance of a hypothetical coarsegrained classifier, output mapped to fine-grained senses, on SENSEVAL English all-words tasks.

ordered in descending order of frequency. Since this sense is not necessarily the same as the original sense of the instance, the accuracy of the finegrained answers will be below $100 \%$.

Figure 1 shows the performance of this transformed fine-grained classifier $(\mathrm{CG})$ for nouns and verbs with SENSEVAL-2 and 3 English all words task data (marked as S2 and S3 respectively), along with the baseline WORDNET first sense (BL), and the best-performer classifiers at each SENSEVAL excercise (CL), SMUaw (Mihalcea, 2002) and GAMBL-AW (Decadt et al., 2004) respectively.

There is a considerable difference in terms of improvement over baseline, between the state-of-theart systems and the hypothetical optimal coarsegrained system. This shows us that there is an improvement in performance that we can attain over the state-of-the-art, if we can create a classifier for even a very coarse level of senses, with sufficiently high accuracy. We believe that the chances for such a high accuracy in a coarse-grained sense classifier is better, for several reasons:

- previously reported good performance for coarse grained systems (Yarowsky, 1992)

- better availability of data, due to the possibility of reusing data created for different words. For instance, labelled data for the noun 'crane' is not found in SEMCOR corpus at all, but there are more than 1000 sample instances for the concept ANIMAL, and more than 9000 for ARTEFACT. 
- higher inter-annotator agreement levels and lower corpus/genre dependencies in training/testing data due to coarser senses.

\subsection{Overall approach}

Basically, we assume that we can learn the 'concepts', in terms of WORDNET unique beginners, using a set of data labelled with these concepts, regardless of the actual word that is labelled. Hence, we can use a generic data set that is large enough, where various words provide training examples for these concepts, instead of relying upon data from the examples of the same word that is being classified.

Unfortunately, simply labelling each instance with its semantic class and then using standard supervised learning algorithms did not work well. This is probably because the effectiveness of the feature patterns often depend on the actual word being disambiguated and not just its semantic class. For example, the phrase 'run the newspaper' effectively indicates that 'newspaper' belongs to the semantic class GROUP. But 'run the tape' indicates that 'tape' belongs to the semantic class ARTEFACT. The collocation 'run the' is effective for indicating the GROUP sense only for 'newspaper' and closely related words such as 'department' or 'school'.

In this experiment, we use a k-nearest neighbor classifier. In order to allow training examples of different words from the same semantic class to effectively provide information for each other, we modify the distance between instances in a way that makes the distance between instances of similar words smaller. This is described in Section 3.

The rest of the paper is organized as follows: In section 2, we discuss several related work. We proceed on to a detailed description of our system in section 3, and discuss the empirical results in section 4 , showing that our representation can yield state of the art performance.

\section{Related Work}

Using generic classes as word senses has been done several times in WSD, in various contexts. Resnik (1997) described a method to acquire a set of conceptual classes for word senses, employing selectional preferences, based on the idea that certain linguistic predicates constraint the semantic interpretation of underlying words into certain classes.
The method he proposed could acquire these constraints from a raw corpus automatically.

Classification proposed by Levin (1993) for English verbs remains a matter of interest. Although these classes are based on syntactic properties unlike those in WORDNET, it has been shown that they can be used in automatic classifications (Stevenson and Merlo, 2000). Korhonen (2002) proposed a method for mapping WORDNET entries into Levin classes.

WSD System presented by Crestan et al. (2001) in SENSEVAL-2 classified words into WORDNET unique beginners. However, their approach did not use the fact that the primes are common for words, and training data can hence be reused.

Yarowsky (1992) used Roget's Thesaurus categories as classes for word senses. These classes differ from those mentioned above, by the fact that they are based on topical context rather than syntax or grammar.

\section{Basic Design of the System}

The system consists of three classifiers, built using local context, part of speech and syntax-based relationships respectively, and combined with the mostfrequent sense classifier by using weighted majority voting. Our experiments (section 4.3) show that building separate classifiers from different subsets of features and combining them works better than building one classifier by concatenating the features together.

For training and testing, we used publicly available data sets, namely SEMCOR corpus (Miller et al., 1993) and SENSEVAL English all-words task data. In order to evaluate the systems performance in vivo, we mapped the outputs of our classifier to the answers given in the key. Although we face a penalty here due to the loss of granularity, this approach allows a direct comparison of actual usability of our system.

\subsection{Data}

As training corpus, we used Brown-1 and Brown2 parts of SEMCOR corpus; these parts have all of their open-class words tagged with corresponding WORDNET senses. A part of the training corpus was set aside as the development corpus. This part was selected by randomly selecting a portion of multi- 
class words (600 instances for each part of speech) from the training data set. As labels, the semantic class (lexicographic file number) was extracted from the sense key of each instance. Testing data sets from Senseval-2 and Senseval-3 English all-words tasks were used as testing corpora.

\subsection{Features}

The feature set we selected was fairly simple; As we understood from our initial experiments, widewindow context features and topical context were not of much use for learning semantic classes from a multi-word training data set. Instead of generalizing, wider context windows add to noise, as seen from validation experiments with held-out data.

Following are the features we used:

\subsubsection{Local context}

This is a window of $n$ words to the left, and $n$ words to the right, where $n \in\{1,2,3\}$ is a parameter we selected via cross validation. ${ }^{1}$

Punctuation marks were removed and all words were converted into lower case. The feature vector was calculated the same way for both nouns and verbs. The window did not exceed the boundaries of a sentence; when there were not enough words to either side of the word within the window, the value NULL was used to fill the remaining positions.

For instance, for the noun 'companion' in sentence (given with POS tags)

\section{'Henry/NNP peered/VBD doubtfully/RB at/IN his/PRP\$ drinking/NN compan- ion/NN through/IN bleary/JJ ,/, tear- filled/JJ eyes/NNS ./'}

the local context feature vector is [at, his, drinking, through, bleary, tear-filled], for window size $n=3$. Notice that we did not consider the hyphenated words as two words, when the data files had them annotated as a single token.

\subsubsection{Part of speech}

This consists of parts of speech for a window of $n$ words to both sides of word (excluding the word

\footnotetext{
${ }^{1}$ Validation results showed that a window of two words to both sides yields the best performance for both local context and POS features. $n=2$ is the size we used in actual evaluation.
}

\begin{tabular}{|c|c|c|}
\hline Feature & Example & Value \\
\hline \multicolumn{3}{|c|}{ nouns } \\
\hline Subject - verb & [art] represents a culture & represent \\
\hline Verb - object & He sells his [art] & sell \\
\hline Adjectival modifiers & the ancient [art] of runes & ancient \\
\hline Prepositional connectors & academy of folk [art] & academy of \\
\hline Post-nominal modifiers & the [art] of fishing & of fishing \\
\hline \multicolumn{3}{|c|}{ verbs } \\
\hline Subject - verb & $\mathrm{He}$ [sells] his art & he \\
\hline Verb - object & $\mathrm{He}$ [sells] his art & art \\
\hline Infinitive connector & He will [sell] his art & he \\
\hline Adverbial modifier & He can [paint] well & well \\
\hline Words in split infinitives & to boldly [go] & boldly \\
\hline
\end{tabular}

Table 1: Syntactic relations used as features. The target word is shown inside [brackets]

itself), with quotation signs and punctuation marks ignored. For SEMCOR files, existing parts of speech were used; for SENSEVAL data files, parts of speech from the accompanying Penn-Treebank parsed data files were aligned with the XML data files. The value vector is calculated the same way as the local context, with the same constraint on sentence boundaries, replacing vacancies with NULL.

As an example, for the sentence we used in the previous example, the part-of-speech vector with context size $n=3$ for the verb peered is [NULL, NULL, NNP, RB, IN, PRP\$].

\subsubsection{Syntactic relations with the word}

The words that hold several kinds of syntactic relations with the word under consideration were selected. We used Link Grammar parser due to Sleator and Temperley (1991) because of the informationrich parse results produced by it.

Sentences in SEMCOR corpus files and the SENSEVAL files were parsed with Link parser, and words were aligned with links. A given instance of a word can have more than one syntactic features present. Each of these features was considered as a binary feature, and a vector of binary values was constructed, of which each element denoted a unique feature found in the test set of the word.

Each syntactic pattern feature falls into either of two types collocation or relation:

Collocation features Collocation features are such features that connect the word under consideration to another word, with a preposition or an infinitive in between - for instance, the phrase 'art of change-ringing' for the word art. For these features, the feature value consists of two words, which are connected to the given word either from left or 
from right, in a given order. For the above example, the feature value is [ . of . change-ringing], where $\sim$ denotes the placeholder for word under consideration.

Relational features Relational features represent more direct grammatical relationships, such as subject-verb or noun-adjective, the word under consideration has with surrounding words. When encoding the feature value, we specified the relation type and the value of the feature in the given instance. For instance, in the phrase 'Henry peered doubtfully', the adverbial modifier feature for the verb 'peered' is encoded as [adverb-mod doubtfully].

A description of the relations for each part of speech is given in the table 1 .

\subsection{Classifier and instance weighting}

The classifier we used was TiMBL, a memory based learner due to Daelemans et al. (2003). One reason for this choice was that memory based learning has shown to perform well in previous word sense disambiguation tasks, including some best performers in Senseval, such as (Hoste et al., 2001; Decadt et al., 2004; Mihalcea and Faruque, 2004). Another reason is that TiMBL supported exemplar weights, a necessary feature for our system for the reasons we describe in the next section.

One of the salient features of our system is that it does not consider every example to be equally important. Due to the fact that training instances from different instances can provide confusing examples, as shown in section 1.3, such an approach cannot be trusted to give good performance; we verified this by our own findings through empirical evaluations as shown in section 4.2.

\subsubsection{Weighting instances with similarity}

We use a similarity based measure to assign weights to training examples. In the method we use, these weights are used to adjust the distances between the test instance and the example instances. The distances are adjusted according to the formula

$$
\Delta^{E}(X, Y)=\frac{\Delta(X, Y)}{e w_{X}+\epsilon},
$$

where $\Delta^{E}(X, Y)$ is the adjusted distance between instance $Y$ and example $X, \Delta(X, Y)$ is the original distance, $e w_{X}$ is the exemplar weight of instance $X$. The small constant $\epsilon$ is added to avoid division by zero.

There are various schemes used to measure intersense similarity. Our experiments showed that the measure defined by Jiang and Conrath (1997) (JCn) yields best results. Results for various weighting schemes are discussed in section 4.2.

\subsubsection{Instance weighting explained}

The exemplar weights were derived from the following method:

1. pick a labelled example $e$, and extract its sense $s_{e}$ and semantic class $c_{e}$.

2. if the class $c_{e}$ is a candidate for the current test word $w$, i.e. $w$ has any senses that fall into $c_{e}$, find out the most frequent sense of $w, s_{w}^{c e}$, within $c_{e}$. We define the most frequent sense within a class as the sense that has the lowest WORDNET sense number within that class. If none of the senses of $w$ fall into $c_{e}$, we ignore that example.

3. calculate the relatedness measure between $s_{e}$ and $s_{w}^{c e}$, using whatever the similarity metric being considered. This is the exemplar weight for example $e$.

In the implementation, we used freely available WordNet: : Similarity package (Pedersen et al., 2004). ${ }^{2}$

\subsection{Classifier optimization}

A part of SEMCOR corpus was used as a validation set (see section 3.1). The rest was used as training data in validation phase. In the preliminary experiments, it was seen that the generally recommended classifier options yield good enough performance, although variations of switches could improve performance slightly in certain cases. Classifier options were selected by a search over the available option space for only three basic classifier parameters, namely, number of nearest neighbors, distance metric and feature weighting scheme.

\footnotetext{
${ }^{2}$ WordNet: :Similarity is a perl package available freely under GNU General Public Licence. http://wnsimilarity.sourceforge.net.
} 


\begin{tabular}{|l|r|r|}
\hline Classifier & Senseval-2 & Senseval-3 \\
\hline Baseline & 0.617 & 0.627 \\
POS & 0.616 & 0.614 \\
Local context & 0.627 & 0.633 \\
Synt. Pat & 0.620 & 0.612 \\
Concatenated & 0.609 & 0.611 \\
Combined & $\mathbf{0 . 6 3 1}$ & $\mathbf{0 . 6 4 3}$ \\
\hline
\end{tabular}

Table 2: Results of baseline, individual, and combined classifiers: recall measures for nouns and verbs combined.

\section{Results}

In what follows, we present the results of our experiments in various test cases. ${ }^{3}$ We combined the three classifiers and the WORDNET first-sense classifier through simple majority voting. For evaluating the systems with SENSEVAL data sets, we mapped the outputs of our classifiers to WORDNET senses by picking the most-frequent sense (the one with the lowest sense number) within each of the class. This mapping was used in all tests. For all evaluations, we used SENSEVAL official scorer.

We could use the setting only for nouns and verbs, because the similarity measures we used were not defined for adjectives or adverbs, due to the fact that hypernyms are not defined for these two parts of speech. So we list the initial results only for nouns and verbs.

\subsection{Individual classifiers vs. combination}

We evaluated the results of the individual classifiers before combination. Only local context classifier could outperform the baseline in general, although there is a slight improvement with the syntactic pattern classifier on SENSEVAL-2 data.

The results are given in the table 2, together with the results of voted combination, and baseline WORDNET first sense. Classifier shown as 'concatenated' is a single classifier trained from all of these feature vectors concatenated to make a single vector. Concatenating features this way does not seem to improve performance. Although exact reasons for this are not clear, this is consistent with pre-

\footnotetext{
${ }^{3}$ Note that the experiments and results are reported for SENSEVAL data for comparison purposes, and were not involved in parameter optimization, which was done with the development sample.
}

\begin{tabular}{|l|r|r|}
\hline & Senseval-2 & Senseval-3 \\
\hline No similarity used & 0.608 & 0.599 \\
Resnik & 0.540 & 0.522 \\
JCn & $\mathbf{0 . 6 3 1}$ & $\mathbf{0 . 6 4 3}$ \\
\hline
\end{tabular}

Table 3: Effect of different similarity schemes on recall, combined results for nouns and verbs

\begin{tabular}{|l|r|r|}
\hline & Senseval-2 & Senseval-3 \\
\hline SM & 0.631 & 0.643 \\
GW & 0.634 & 0.649 \\
LW & $\mathbf{0 . 6 4 1}$ & $\mathbf{0 . 6 5 0}$ \\
\hline
\end{tabular}

Table 4: Improvement of performance with classifier weighting. Combined results for nouns and verbs with voting schemes Simple Majority (SM), Global classifier weights $(\mathrm{GW})$ and local weights (LW).

vious observations (Hoste et al., 2001; Decadt et al., 2004) that combining classifiers, each using different features, can yield good performance.

\subsection{Effect of similarity measure}

Table 3 shows the effect of JCn and Resnik similarity measures, along with no similarity weighting, for the combined classifier. It is clear that proper similarity measure has a major impact on the performance, with Resnik measure performing worse than the baseline.

\subsection{Optimizing the voting process}

Several voting schemes were tried for combining classifiers. Simple majority voting improves performance over baseline. However, previously reported results such as (Hoste et al., 2001) and (Decadt et al., 2004) have shown that optimizing the voting process helps improve the results. We used a variation of Weighted Majority Algorithm (Littlestone and Warmuth, 1994). The original algorithm was formulated for binary classification tasks; however, our use of it for multi-class case proved to be successful.

We used the held-out development data set for adjusting classifier weights. Originally, all classifiers have the same weight of 1 . With each test instance, the classifier builds the final output considering the weights. If this output turns out to be wrong, the classifiers that contributed to the wrong answer get their weights reduced by some factor. We could ad- 


\begin{tabular}{|l|r|r|}
\hline & Senseval-2 & Senseval-3 \\
\hline System & 0.777 & 0.806 \\
Baseline & 0.756 & 0.783 \\
\hline
\end{tabular}

Table 5: Coarse grained results

just the weights locally or globally; In global setting, the weights were adjusted using a random sample of held-out data, which contained different words. These weights were used for classifying all words in the actual test set. In local setting, each classifier weight setting was optimized for individual words that were present in test sets, by picking up random samples of the same word from SEMCOR. ${ }^{4}$ Table 4 shows the improvements with each setting.

Coarse grained (at semantic-class level) results for the same system are shown in table 5. Baseline figures reported are for the most-frequent class.

\subsection{Final results on SENSEVAL data}

Here, we list the performance of the system with adjectives and adverbs added for the ease of comparison. Due to the facts mentioned at the beginning of this section, our system was not applicable for these parts of speech, and we classified all instances of these two POS types with their most frequent sense. We also identified the multi-word phrases from the test documents. These phrases generally have a unique sense in WORDNET ; we marked all of them with their first sense without classifying them. All the multiple-class instances of nouns and verbs were classified and converted to WORDNET senses by the method described above, with locally optimized classifier voting.

The results of the systems are shown in tables 7 and 8. Our system's results in both cases are listed as Simil-Prime, along with the baseline WORDNET first sense (including multi-word phrases and ' $U$ ' answers), and the two best performers' results reported. ${ }^{5}$ These results compare favorably with the official results reported in both tasks.

\footnotetext{
${ }^{4}$ Words for which there were no samples in SEMCOR were classified using a weight of 1 for all classifiers.

${ }^{5}$ The differences of the baseline figures from the previously reported figures are clearly due to different handling of multiword phrases, hyphenated words, and unknown words in each system. We observed by analyzing the answer keys that even better baseline figures are technically possible, with better techniques to identify these special cases.
}

\begin{tabular}{|l|r|r|}
\hline & Senseval-2 & Senseval-3 \\
\hline Micro Average & $<0.0001$ & $<0.0001$ \\
Macro Average & 0.0073 & 0.0252 \\
\hline
\end{tabular}

Table 6: One tailed paired t-test significance levels of results: $P(T \leqslant t)$

\begin{tabular}{|l|c|}
\hline System & Recall \\
\hline SMUaw (Mihalcea, 2002) & 0.690 \\
Simil-Prime & 0.664 \\
Baseline (WORDNET first sense) & 0.648 \\
CNTS-Antwerp (Hoste et al., 2001) & 0.636 \\
\hline
\end{tabular}

Table 7: Results for SENSEVAL-2 English all words data for all parts of speech and fine grained scoring.

Significance of results To verify the significance of these results, we used one-tailed paired t-test, using results of baseline WORDNET first sense and our system as pairs. Tests were done both at microaverage level and macro-average level, (considering test data set as a whole and considering per-word average). Null hypothesis was that there is no significant improvement over the baseline. Both settings yield good significance levels, as shown in table 6 .

\section{Conclusion and Future Work}

We analyzed the problem of Knowledge Acquisition Bottleneck in WSD, proposed using a general set of semantic classes as a trade-off, and discussed why such a system is promising. Our formulation allowed us to use training examples from words different from the actual word being classified. This makes the available labelled data reusable for different words, relieving the above problem. In order to facilitate learning, we introduced a technique based on word sense similarity.

The generic classes we learned can be mapped to

\begin{tabular}{|l|c|}
\hline System & Recall \\
\hline Simil-Prime & 0.661 \\
GAMBL-AW-S (Decadt et al., 2004) & 0.652 \\
SenseLearner (Mihalcea and Faruque, 2004) & 0.646 \\
Baseline (WORDNET first sense) & 0.642 \\
\hline
\end{tabular}

Table 8: Results for SENSEVAL-3 English all words data for all parts of speech and fine grained scoring. 
finer grained senses with simple heuristics. Through empirical findings, we showed that our system can attain state of the art performance, when applied to standard fine-grained WSD evaluation tasks.

In the future, we hope to improve on these results: Instead of using WORDNET unique beginners, using more natural semantic classes based on word usage would possibly improve the accuracy, and finding such classes would be a worthwhile area of research. As seen from our results, selecting correct similarity measure has an impact on the final outcome. We hope to work on similarity measures that are more applicable in our task.

\section{Acknowledgements}

Authors wish to thank the three anonymous reviewers for their helpful suggestions and comments.

\section{References}

E. Crestan, M. El-Bèze, and C. De Loupy. 2001. Improving wsd with multi-level view of context monitored by similarity measure. In Proceeding of SENSEVAL-2: Second International Workshop on Evaluating Word Sense Disambiguation Systems, Toulouse, France.

Walter Daelemans, Jakub Zavrel, Ko van der Sloot, and Antal van den Bosch. 2003. TiMBL: Tilburg Memory Based Learner, version 5.0, reference guide. Technical report, ILK 03-10.

Bart Decadt, Véronique Hoste, Walter Daelemans, and Antal Van den Bosch. 2004. GAMBL, genetic algorithm optimization of memory-based wsd. In Senseval-3: Third Intl. Workshop on the Evaluation of Systems for the Semantic Analysis of Text.

P. Edmonds and S. Cotton. 2001. Senseval-2: Overview. In Proc. of the Second Intl. Workshop on Evaluating Word Sense Disambiguation Systems (Senseval-2).

C. Fellbaum. 1998. WordNet: An Electronic Lexical Database. The MIT Press, Cambridge, MA.

Véronique Hoste, Anne Kool, and Walter Daelmans. 2001. Classifier optimization and combination in English all words task. In Proceeding of SENSEVAL-2: Second International Workshop on Evaluating Word Sense Disambiguation Systems.

J. Jiang and D. Conrath. 1997. Semantic similarity based on corpus statistics and lexical taxonomy. In Proceedings of International Conference on Research in Computational Linguistics.
Anna Korhonen. 2002. Assigning verbs to semantic classes via wordnet. In Proceedings of the COLING Workshop on Building and Using Semantic Networks.

Beth Levin. 1993. English Verb Classes and Alternations. University of Chicago Press, Chicago, IL.

N Littlestone and M.K. Warmuth. 1994. The weighted majority algorithm. Information and Computation, 108(2):212-261.

Rada Mihalcea and Tim Chklovski. 2003. Open Mind Word Expert: Creating large annotated data collections with web users' help. In Proceedings of the EACL 2003 Workshop on Linguistically Annotated Corpora.

Rada Mihalcea and Ehsanul Faruque. 2004. Senselearner: Minimally supervised word sense disambiguation for all words in open text. In Senseval-3: Third Intl. Workshop on the Evaluation of Systems for the Semantic Analysis of Text.

Rada Mihalcea. 2002. Bootstrapping large sense tagged corpora. In Proc. of the 3rd Intl. Conference on Languages Resources and Evaluations.

G. Miller, C. Leacock, T. Randee, and R. Bunker. 1993. A semantic concordance. In Proc. of the 3rd DARPA Workshop on Human Language Technology.

Hwee Tou Ng. 1997. Getting serious about word sense disambiguation. In Proceedings of the ACL SIGLEX Workshop on Tagging Text with Lexical Semantics: Why, What, and How?, pages 1-7.

T. Pedersen, S. Patwardhan, and J. Michelizzi. 2004. Wordnet::Similarity - Measuring the relatedness of concepts. In Proceedings of the Nineteenth National Conference on Artificial Intelligence (AAAI-04).

P. Resnik. 1997. Selectional preference and sense disambiguation. In Proc. of ACL Siglex Workshop on Tagging Text with Lexical Semantics, Why, What and How?

D. Sleator and D. Temperley. 1991. Parsing English with a Link Grammar. Technical report, Carnegie Mellon University Computer Science CMU-CS-91-196.

B. Snyder and M. Palmer. 2004. The English all-words task. In Senseval-3: Third Intl. Workshop on the Evaluation of Systems for the Semantic Analysis of Text.

Suzanne Stevenson and Paola Merlo. 2000. Automatic lexical acquisition based on statistical distributions. In Proc. of the 17th conf. on Computational linguistics.

David Yarowsky. 1992. Word-sense disambiguation using statistical models of Roget's categories trained on large corpora. In Proceedings of COLING-92, pages 454-460. 